Check for updates

Cite this: RSC Adv., 2017, 7, 25796

Received 16th March 2017

Accepted 8th May 2017

DOI: 10.1039/c7ra03127k

rsc.li/rsc-advances

\section{Fabrics coated with hot-iron-treated graphene oxide for a self-cleaning and mechanically robust water-oil separation material $\dagger$}

\author{
Tao Gong, Jongwoon Kim, Ju Yeon Woo, Jin-Hyeok Jang, Seung Eun Lee \\ and Chang-Soo Han (iD *
}

\begin{abstract}
A simple method was reported to fabricate self-cleaning and water-oil separation fabrics sprayed with hotiron-treated graphene oxide (GO). The GO solution was prepared with a modified Hummers' method and coated on the fabrics by spraying or soaking method. A $160{ }^{\circ} \mathrm{C}$ hot iron was pressed at the surface of the fabrics to make it flat, dry, thermally reduced in part, and strongly bonded. Afterward, the fabrics were thermally reduced at $250{ }^{\circ} \mathrm{C}$ for 20 minutes in an oven. The reduced graphene oxide ( $\mathrm{rGO}$ ) coated fabrics exhibited a superhydrophobic nature with a water contact angle of $129.4^{\circ}$, through which water could barely permeate the fabrics, in contrast to oil and organic solvents of low polarity. Additionally, this rGO fabric presented outstanding mechanical properties as well as a reusable stability.
\end{abstract}

\section{Introduction}

Inspired by the intrinsic hydrophobic properties of the lotus petal or leaf and other living things in nature, so-called selfcleaning clothes have long been a dream of human beings. ${ }^{1-3}$ Multifunctional fabrics with a particular wettability have attracted much interest in both industrial applications and fundamental research. Various surface treatment techniques and design strategies have been inspired by many biological systems. ${ }^{4,5}$ Disregarding the superior properties of fabrics, some of the most widely used ones in our daily lives possess inherent features such as being hydrophilic and getting easily dirty, which have confined their wider application, especially in high-tech fields requiring self-cleaning, personal healthcare, and flexible multifunctional fabrics. ${ }^{6}$ To date, there have been relatively few prior studies in the area of hydrophobic coating surfaces. Sparks et al. reported a method for the fabrication of hydrophobic inorganic-organic thiolene coating via sequential spraydeposition and photo polymerization under ambient conditions. The spray-deposition process and nanoparticle agglomeration/dispersion provide surfaces with hierarchical morphologies exhibiting both micro- and nanoscale roughness. ${ }^{7}$ Kim et al. used PTFE- $\mathrm{TiO}_{2}$ nanocomposite films with superhydrophobic and superhydrophilic properties, deposited by a supersonic spray coating technique. Wettability tuning was achieved by simply varying the relative ratio of PTFE and $\mathrm{TiO}_{2}{ }^{8}$ Tian et al. suggested PS-SFVS to study hydrophobic interfaces

School of Mechanical Engineering, Korea University, Anam, Seongbuk, Seoul 02841, Republic of Korea. E-mail: cshan@korea.ac.kr

$\dagger$ Electronic supplementary information (ESI) available. See DOI: $10.1039 / \mathrm{c} 7 \mathrm{ra} 03127 \mathrm{k}$ and monitored the presence of excess ions at the surface. ${ }^{9}$ Wang et al. fabricated bio-inspired hydrophobic surfaces via an electrospinning technique and demonstrated the possibility of optical device application. ${ }^{10}$ Deng et al. designed a porous deposition of candle soot coated with a 25 nanometer-thick silica shell. A black coating became transparent after calcination at $600{ }^{\circ} \mathrm{C} .{ }^{11}$ However, up to now, most hydrophobic fabrics are produced by sophisticated processes need expensive equipment and/or rigid conditions to modify surface properties. ${ }^{7}$ In addition, such surface textures are highly susceptible to mechanical wear and abrasion may transform the surface chemistry as well. ${ }^{12}$ Invasion could lead to a loss of liquid repellency, which makes mechanical durability a central concern for practical applications. ${ }^{13}$ Besides, various other problems still exist, such as the raw materials' high cost, weak mechanical stability, and difficulties of scalable production. ${ }^{14,15}$ Thus, a straightforward approach of producing hydrophobic fabrics under a wide range of conditions, such as robust mechanical as well as electrical properties and water-oil separation is direly needed. A key factor in the surface wettability transition is the coating material. In recent years, there has been an increasing interest of researchers in graphene oxide (GO) because of rich oxygen-containing functional groups on its basal planes and along its sheet edges ${ }^{16}$ which have a high affinity with water molecules, so GO is hydrophilic and can easily be dispersed in water. ${ }^{17}$

In this study, we introduced a simple and low-cost method to develop self-cleaning and water-oil separation fabrics, which can easily pass the oil but selectively reject water. To this end, we newly developed a mechanical and thermal iron pressing (MTIP) process to make the fabric mechanically durable and added a high-temperature thermal reduction process to complete the coating process. As the result, we were able to 
fabricate multifunctional reduced graphene oxide (rGO) coated fabrics with water repellency and oil separation features.

\section{Experimental}

\section{Materials and methods}

Preparation of the GO solution. The GO solution was chemically synthesized from natural graphite flakes by using a modified Hummer method. Twelve g of graphite (Alfa Aesar, $99.8 \%, 325 \mathrm{mesh}$ ), $740 \mathrm{~mL}$ of $\mathrm{H}_{2} \mathrm{SO}_{4}$ (purity 95\%), and $10 \mathrm{~g}$ of $\mathrm{NaNO}_{3}$ were added to a beaker alongside a magnetic stirrer. $\mathrm{KMnO}_{4}$ in the amount of $48 \mathrm{~g}$ was gradually added into this mixture subsequent to one hour of cooling in an ice water bath. The mixture was stirred until it became a greatly adhesive liquid at room temperature and $1200 \mathrm{~mL}$ of $5 \mathrm{wt} \% \mathrm{H}_{2} \mathrm{SO}_{4}$ aqueous solution was added, which was then stirred for another hour. Later, $30 \mathrm{wt} \% \mathrm{H}_{2} \mathrm{O}_{2}$ aqueous solution was gently added until the mixture became yellowish, which was then stirred for one hour. The products were rinsed more than 15 times first by using a mixture of $5 \mathrm{wt} \% \mathrm{H}_{2} \mathrm{O}_{2}$ and $3 \mathrm{wt} \% \mathrm{H}_{2} \mathrm{SO}_{4}$ and then with DI water until neutral to obtain the GO solution which had a concentration of approximately $0.8 \mathrm{mg} \mathrm{mL} \mathrm{m}^{-1}$. This aqueous solution was sonicated for 5-10 minutes until the GO was separated in the DI water into individual sheets with an average lateral dimension of more than one $\mu \mathrm{m}$ and remained stable in vials for more than six months.

Preparation of the fabrics coated with rGO. The fabrics were sprayed by GO solutions with a concentration of $0.8 \mathrm{mg} \mathrm{mL}{ }^{-1}$. Afterwards, the wet fabrics were pressed by a hot iron $\left(160^{\circ} \mathrm{C}\right)$ with $1.5 \mathrm{~kg}$ above their own weight, which flattened, dried, and partially reduced them. The whole thermal reduction process lasted 20 to 40 minutes, depending on the fabrics' area. In the initial state, the GO flakes coated very rapidly on the hydrophilic fabrics. The surface structure of the rGO top layer is the wettability key point.

\section{Characterization}

Scanning electron microscope (SEM). To check the topographies of the pristine and prepared fabrics, $1 \mathrm{~nm}$ thick platinum was deposited on the samples before taking the SEM measurement.

Raman spectroscopy. A Raman spectrometer (LabRam ARAMIS IR2) equipped with a $532 \mathrm{~nm}$ laser line and a $\times 100$ objective was used. The backscattered Raman light was diffracted by 1800 gratings mm. To avoid local heating effects, the laser power density was kept below $80 \mu \mathrm{W} \mu \mathrm{m}^{-2}$.

X-ray photoelectron spectroscopy (XPS). An XPS system was employed by properly selecting a monochromatic $\mathrm{Al} \mathrm{K} \alpha$ X-ray source (X-Tool, UlVAC-PHI) and adjusting the X-ray's energy. The spot size of each measurement was $0.6 \mu \mathrm{m}^{2}$.

\section{Mechanical and electrical tests}

Fabrics coated with rGO for tensile testing were scissor-cut into rectangular strips with a width of $25 \mathrm{~mm}$ and lengths in the range $70 \mathrm{~mm}$ to ensure less mechanical damage or microdefects at the edges.
The fabrics' mechanical properties were measured with a tensile test device. Uniaxial tensile measurements were carried out under room conditions with a $50 \mathrm{~mm}$ gap between the clamps (Fig. 6a). This will be elaborated in the Results and discussion section.

To check the conductivity of the fabrics coated with rGO and the relationship with the elongation ratio, a multimeter was used to measure the resistance from one end of the fabrics to the other during elongation by the tensile test device.

\section{Results and discussion}

GO materials have already been commercialized and can be purchased from chemical companies. Furthermore, GO can also be conveniently synthesized by a modified Hummer's method in the laboratory. In this study, self-made GO solutions with a concentration of $0.8 \mathrm{mg} \mathrm{mL}^{-1}$ were used as the spraying material without further modification..$^{18}$ Only a small amount of the GO aqueous solution was required for our research, depending on the fabrics' sizes.

A spray coating method rather than a soaking method was used to maintain a uniform GO distribution on the substrate and to precisely control the coating thickness. Since fabrics are composed of ether, alcohol, and other functional groups, GO also contains polar functional groups such as $-\mathrm{COOH},-\mathrm{OH}$, and epoxy groups. ${ }^{19,20}$ A hydrogen bond electrostatically attracts two polar groups that occurs when a hydrogen $(\mathrm{H})$ atom covalently bounds to a highly electronegative atom such as nitrogen $(\mathrm{N})$, oxygen (O), or fluorine (F) with an electrostatic field of another highly electronegative atom nearby. Hydrogen bonds can also emerge between molecules (intermolecular) or within different parts of a single molecule (intramolecular). XPS deconvoluted spectrums of O1s and C1s (Fig. 4) show that fabrics are composed of cellulose fiber with ether and hydroxyl groups. GO also contains active lone pairs and polar functional groups such as $-\mathrm{COOH},-\mathrm{OH}$, and epoxy, which cause fabrics to interact with GO sheets through hydrogen bonding. On the other hand, the adhesion between the fabrics and the GO sheets was enhanced by the MTIP process based on van der Waals forces. In our experiments, the control groups were set to demonstrate the roles of mechanical press and thermal treatment of the iron (Fig. S1†). One of the fabrics in the control group was just spraying with the GO solution without iron treatment as demonstrated in the process B1. The other fabric was coated with GO solution and iron-pressed at room temperature. As we know, hydrophobic materials repel water and permit oil and low polarity organic solvents to flow through them..$^{21,22}$ Therefore, if the hydrophilic fabrics' surfaces were converted into hydrophobic materials by reduced GO through thermal treatment, they would be endowed with a hydrophobic feature. GO could be reduced with reducing agents such as sodium borohydride, hydrazine, and vitamin $C^{.20,23-25}$ To simplify the preparation process, to further reduce the cost, and to avoid containment by other reducing agents, thermal reduction was chosen in our investigation as the method to achieve rGO. ${ }^{26}$

The simple preparation process of hydrophobic fabrics as well as the surface appearance of pristine and prepared fabrics 
(Fig. 1) were presented by optical and SEM images (Fig. 2) respectively. The pristine fabrics are hydrophilic material with a rough texture and a porous structure which should attest to their potential strong water-absorbing capacity. However, in the prepared fabrics as separation material, the coated ones were covered with hydrophobic rGO sheets due to chemical and hydrogen bonding, which entrust the fabrics with hydrophobic ability. Compared with the Raman spectra measurements of the fabrics coated with rGO and without the MTIP process, fabrics with the MTIP process demonstrated that the relative intensity of the D-band and the G-band decreased (Fig. 3a). ${ }^{27}$ The four attenuated total reflectance Fourier transform infrared spectroscopy (ATR-FTIR) (Fig. 3b) spectra containing oxygen functional groups were largely reduced in the fabrics treated by the MTIP process, indicating that the thermal and mechanical pressing treatment changed the chemical structure of the fabrics coated with GGO $^{28}$ X-ray photoelectron spectroscopy (XPS) was used to further study the changes in the fabrics' elemental composition after thermal treatment (Fig. 4). In the high-resolution XPS spectra, the C1s spectrum for pristine filter fabric demonstrated that it contained six kinds of carbon atoms in different functional groups, namely $\mathrm{C}=\mathrm{C}(284.0 \mathrm{eV}), \mathrm{C}-\mathrm{C}$ (284.6 eV), C-N (286.2 eV), C-O (286.8 eV), carbonyl C $=\mathrm{O}(288.5$ $\mathrm{eV})$, and carboxylate $\mathrm{O}=\mathrm{C}-\mathrm{O}(290.2 \mathrm{eV}) .{ }^{29}$ The water-oil separation fabrics included a higher ratio of oxygen-containing carbon-bond functional groups from GO, carboxyl $\mathrm{C}(\mathrm{O}) \mathrm{O}$. Moreover, the intensity ratio of $\mathrm{C}(\mathrm{O}) / \mathrm{C}-\mathrm{C}$ decreased to 4.15 with an elevation in the thermal reduced temperature at $250{ }^{\circ} \mathrm{C}$. The coated fabrics did not get wet and GO solutions drops formed spheres on it while the uncoated fabric completely wetted and were stained by GO solutions (Fig. S2 $\dagger$ ) which exhibited their superhydrophobic nature with the contact angles $129.4^{\circ}$ after the MTIP process and thermal reduction.

To verify the excellent water-repelling and superoleophilic property with chemical stability, we performed a water-oil separation experiment. Gravity drives this water-oil separation process under ambient pressure. While the water-oil mixture was filtered using the rGO coated fabrics, we chose several types of organic solvents with different densities (chlorobenzene, $\sim 1.1058 \mathrm{~g} \mathrm{~mL}^{-1}$; hexane, $\sim 0.659 \mathrm{~g} \mathrm{~mL}^{-1}$; chloroform, $\sim 1.4788 \mathrm{~g}$
$\mathrm{mL}^{-1}$ ) and mineral oil to check the water-oil separation capacity. Organic solvents or oil were freely drained by the separation fabrics, but water did not pass through (Fig. 5). The fabrics' separation fluxes of various pure organic solvents were measured using a filter apparatus. The area of separation for the fabrics was around $24.8 \mathrm{~cm}^{2}$, the pure organic solvents' volume was $150 \mathrm{~mL}$, and the flux was calculated by the filtration time. The average separation flux of filter fabrics was $420.7 \mathrm{~L} \mathrm{~h}^{-1} \mathrm{~m}^{-2}$. For the separation process of water-oil mixtures in the pyramidshaped separation fabrics (Fig. 5c), the separation flux was calculated by the volume of filtrate per unit time. ${ }^{30}$ The separation fabric had similar average separation flux for the organic solvent-water mixtures $\left(\sim 0.63 \mathrm{~L} \mathrm{~h}^{-1}\right)$ whereas the mineral oilwater mixture had a decreased separation flux $\left(\sim 0.01 \mathrm{~L} \mathrm{~h}^{-1}\right)$. Interestingly, this correlation was related to the liquid viscosity and the porosity of separation fabrics greatly influenced the difference of the separation flux.

To further inspect the separation performance of the prepared fabrics, the separation selectivity was investigated by measuring the weight change of water in water-oil mixture before and after the separation procedure. Water $(30 \mathrm{~mL})$ and 30 $\mathrm{mL}$ oil were used. The grade efficiency with respect to water and oil was above $93 \%$, which demonstrated that the separation fabrics did not permit water to pass through their pores coated with rGO. Besides, there was almost no change regarding the separation selectivity, even though different organic solvents and oil were used. In addition, adsorption was inexorable in the water-oil separation process due to the lipophilicity of the separation fabric. The loss ratio of low boiling point organic solvents ranged from $4.14 \%$ to $7.32 \%$, because of the combined adsorption evaporation effect of organic solvents. Nonetheless, viscous mineral oil had a higher loss ratio (17.51\%), revealing an interaction and that the adsorption between mineral oil and lipophilic separation fabrics were stronger than organic solvents. The results indicated that the separation fabric could be a promising tool to clean up oil-spill containments.

To compare the self-cleaning capability of the fabrics, we poured coffee on the fabric coated with rGO, which formed complete spheres on the substrate without penetrating the surface as well (Fig. S3†). Moreover, when tomato ketchup was

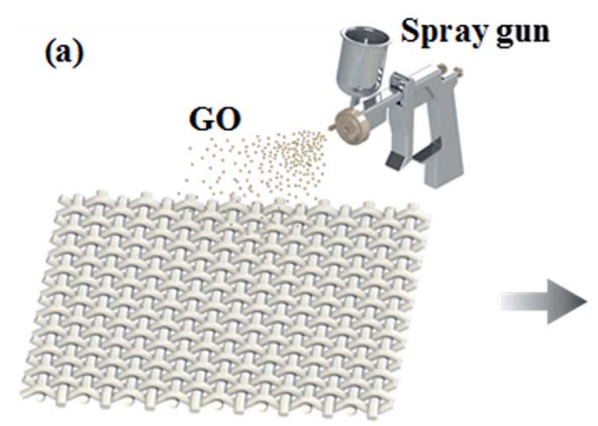

Pristine fabric (b)

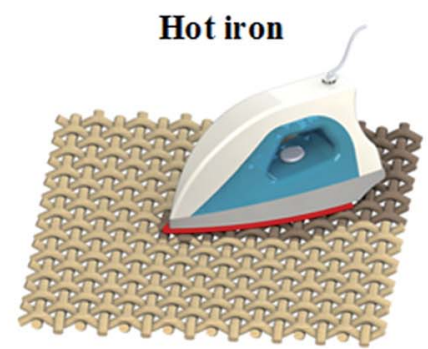

Fabric coated with GO (c)

\section{Heat treatment $250^{\circ} \mathrm{C}$ in the oven for $20 \mathrm{mins}$}

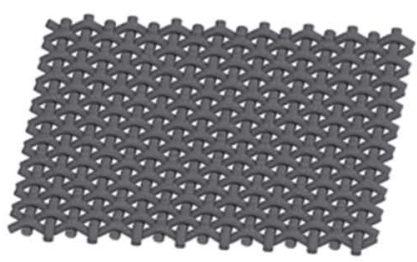

Fabric coated with rGO

Fig. 1 Schematic representation of fabrics coated with $\mathrm{rGO}$ using the MTIP process. (a) The GO solution was sprayed on the pristine fabrics; (b) adhesion between the fabrics and the GO sheets was enhanced and GO was partially reduced by the mechanical and thermal iron pressing (MTIP) process; (c) further thermally reduced fabrics coated with GO in the oven at $250{ }^{\circ} \mathrm{C}$ for 20 minutes. 

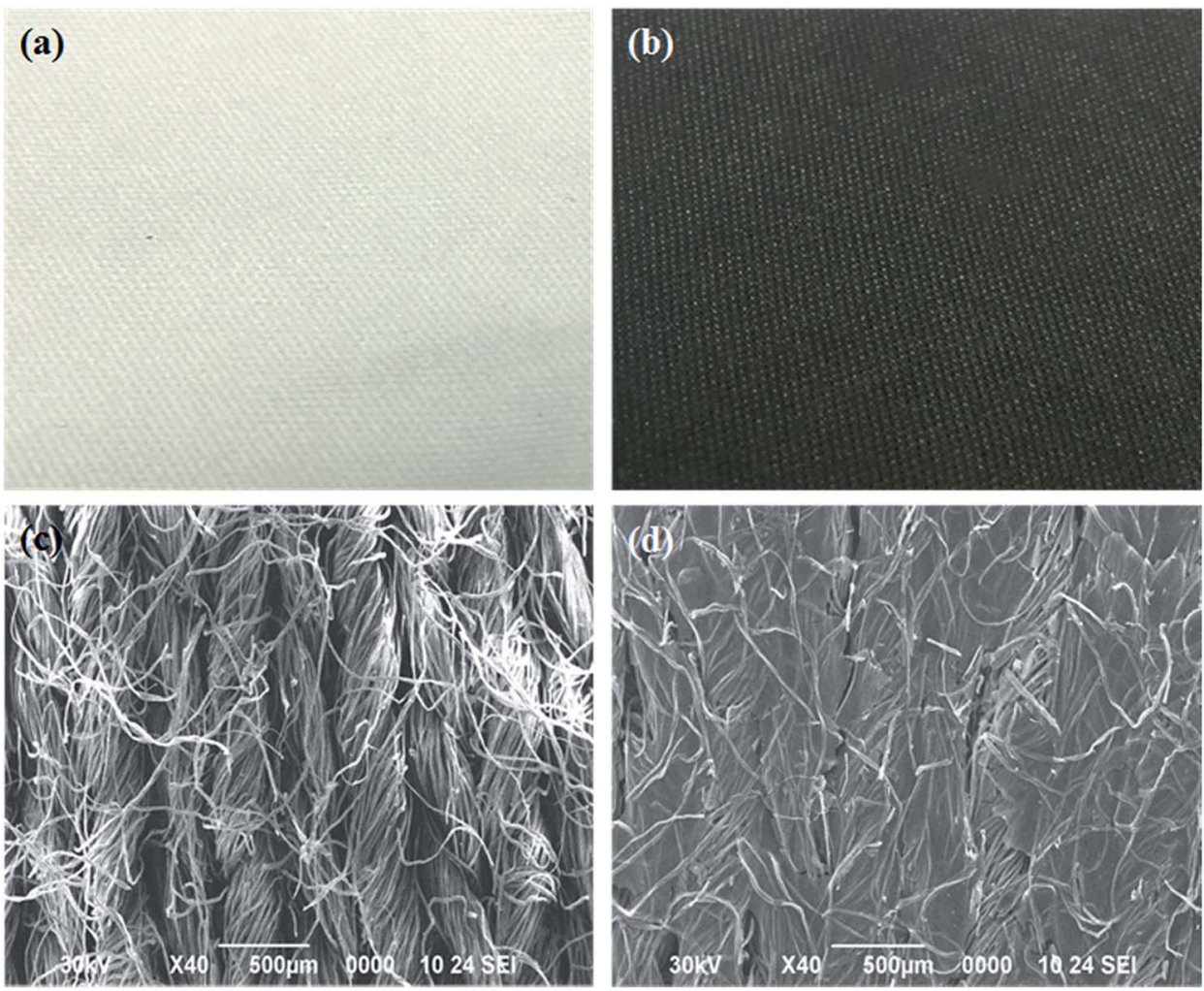

Fig. 2 Photo images of (a) pristine fabrics, (b) rGO-coated fabrics by the MTIP method as well as SEM images of the (c) pristine fabrics, and (d) rGO-coated fabrics by the MTIP method.

(a)

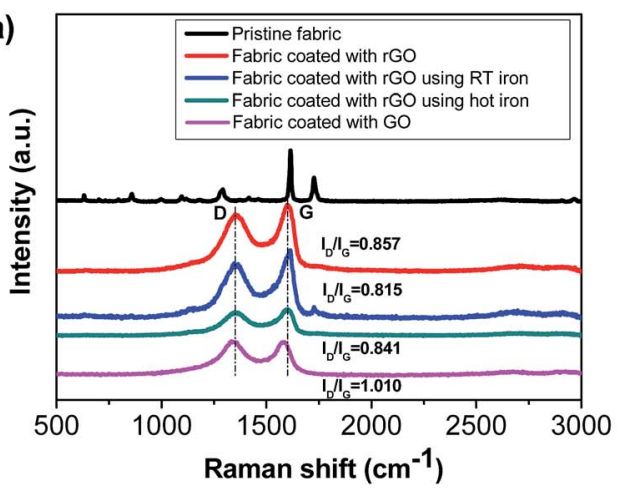

(b)

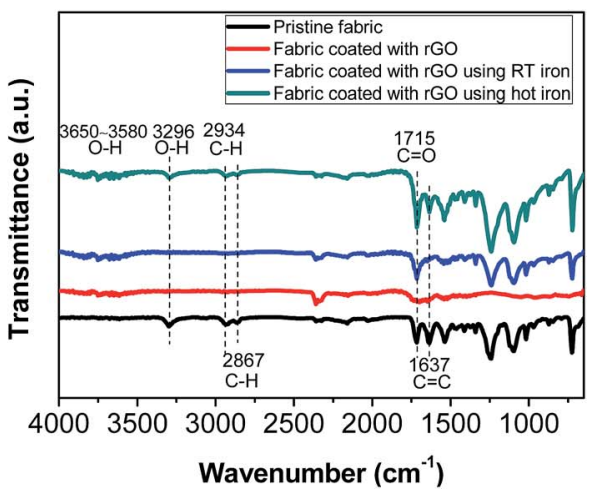

Fig. 3 Characterization of the pristine and prepared fabrics. (a) Raman spectra of the pristine fabrics and rGO-coated fabrics. (b) FTIR spectrums show the functional group in the pristine fabrics and the $\mathrm{rGO}$-coated fabrics.

placed on the rGO-coated fabric and followed by rinsing a small amount of DI water, the ketchup was immediately removed and the fabric remained dirt free (Fig. S4 $\dagger$ ). These observations supported the excellent water repelling, stain-resistant and selfcleaning abilities of fabrics coated with rGO.

The recyclable stability of separation fabrics was another important factor for cost abatement and environmental conservation. In this study, the stability of separation fabrics was determined by elemental analysis (EA). The change in the $\mathrm{C} / \mathrm{O}$ atomic ratio of separation fabrics in the water-oil separation process could be calculated by EA. It was remarkable that the $\mathrm{C} /$ $\mathrm{O}$ atomic ratio of the pure filter fabrics was 1.24 and that of the separation fabrics increased slightly, owning to the coated rGO sheets. Notwithstanding, there was almost no change in the $\mathrm{C} / \mathrm{O}$ atomic ratio in all five water-oil separation experiments, indicating that the separation fabrics' rGO coating and hydrophobicity was very stable and the selectivity of water and flux were not obviously affected by the multiple separation experiments.

Generally, the hydrophobic surface for self-cleaning applications is subjected to mechanical instability and does not function well after scratching or abrasion. To investigate this problem, the robustness of the coating on fabrics was evaluated by an abrasion test using 100 mesh sand paper as a quite harsh abrasion agent (Fig. 6a) and 10 simulated washing cycles as 
(a)

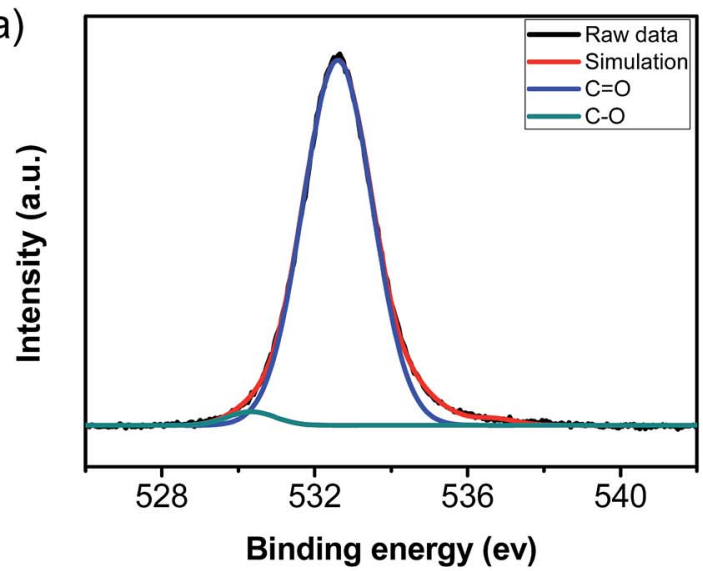

(c)

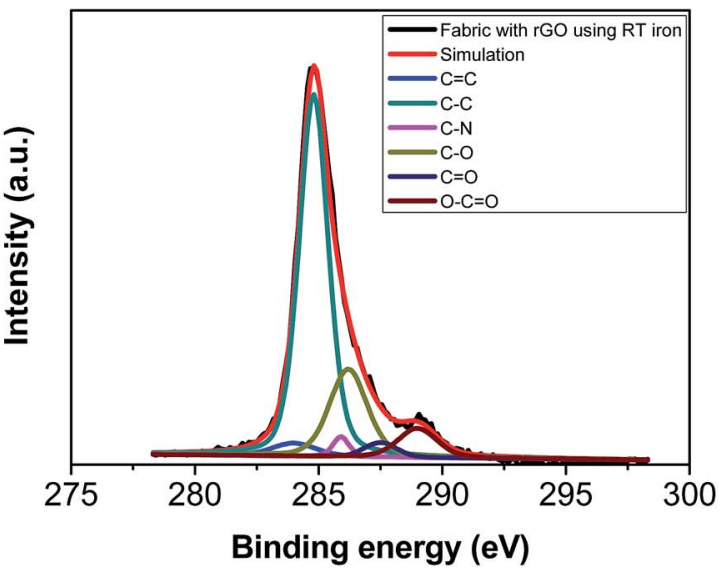

(b)

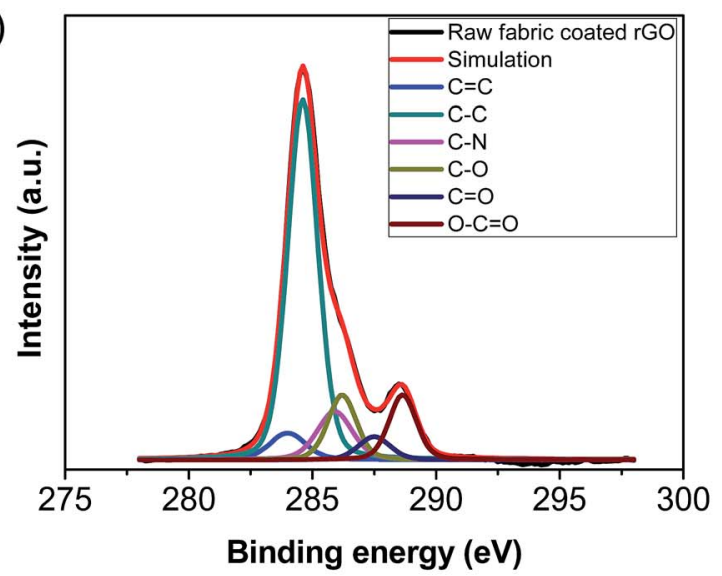

(d)

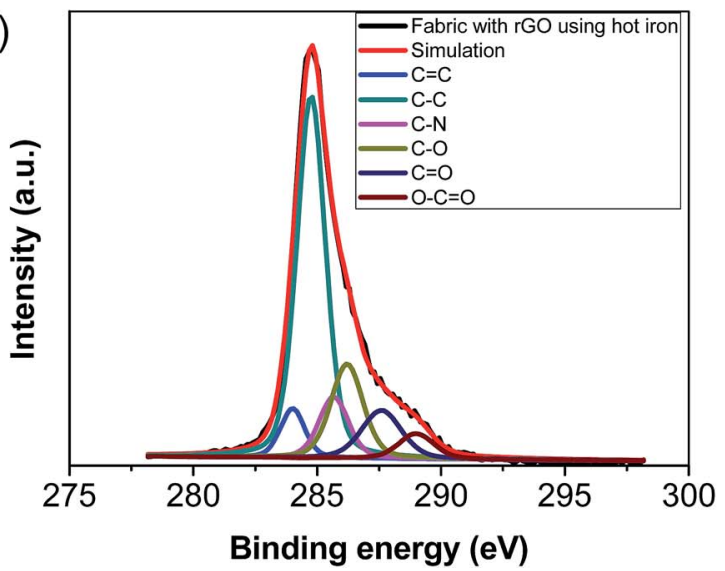

Fig. 4 XPS deconvoluted spectra of O1s (a) rGO-coated fabrics, and spectra of C1s (b) rGO-coated fabric, (c) an rGO-coated fabric with using an iron at room temperature, (d) an rGO-coated fabric using an iron with $160{ }^{\circ} \mathrm{C}$.

(a)
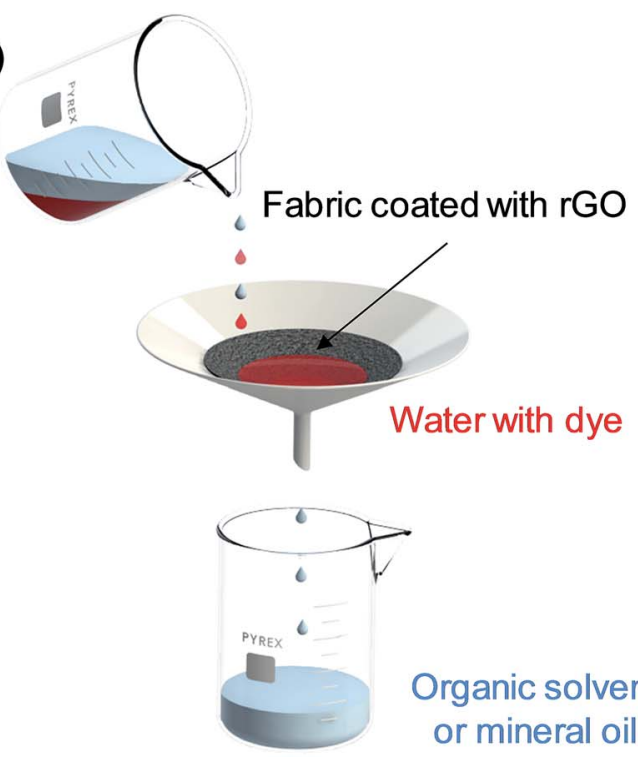

\section{Organic solvent} or mineral oil (b)

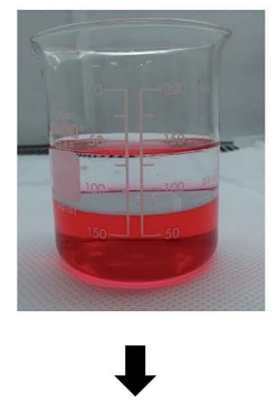

(c)

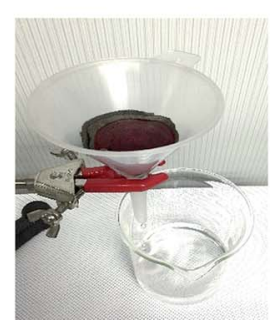

Fig. 5 The water/organic separation experiment using hydrophobic fabrics. The transparent liquid is represented as organic solvents or mineral oil and the red liquid is water with a dye: (a) schematic drawing of the water-oil separation experiment. (b) Mixture of the organic solvent or mineral oil and water with a dye. (c) The water and organic solvent or mineral oil were separated by the rGO-coated fabrics. During separation, only organic solvents or mineral oil passed through the separation fabrics. 
(a)

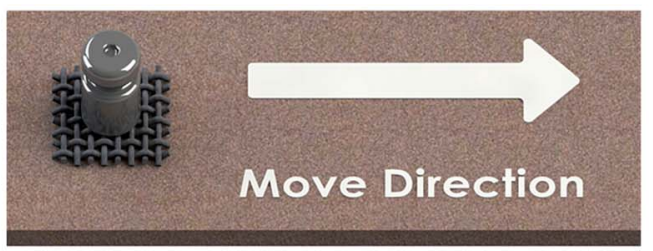

(b)

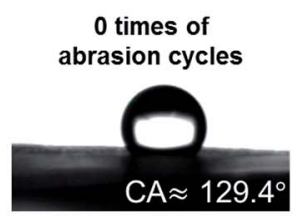

(c)

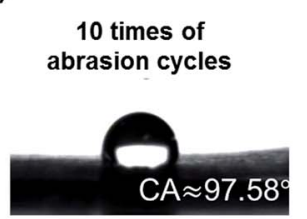

(d)

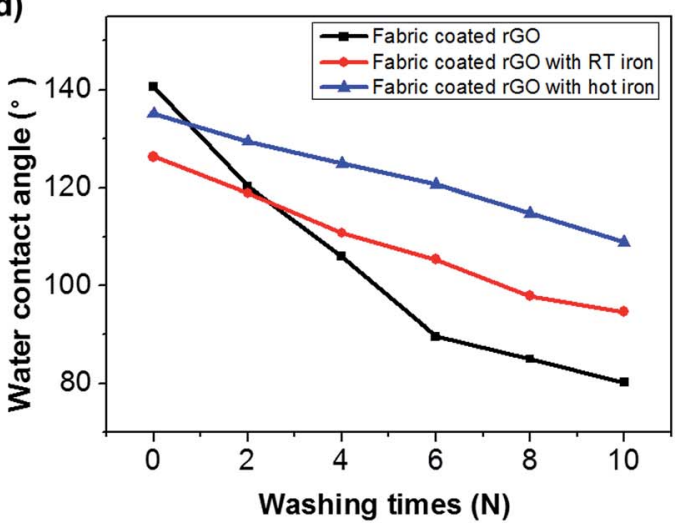

Fig. 6 Water-resistant behavior after sandpaper abrasion and a laundering test. (a) Schematic of a sandpaper abrasion setup; (b) the water contact angle of the rGO-coated fabrics; (c) the water contact angle decreased slightly after the ten abrasion cycles; (d) different treatment methods with their relationships between water contact angles and laundering times.

described in the Experimental section. The WCA change of the abraded surface was measured before and after 10 cycles of abrasions following the similar procedure (Fig. $6 \mathrm{~b}$ and c). It was found that even after 10 times of abrasion, the fabrics coated with rGO were still hydrophobic. When repetitive washings of the several samples were performed, the rGO-coated fabric treated by the MTIP process presented much less degradation than other samples (Fig. 6d). This exhibited an anti-laundering mechanical property. The excellent stability and reusability of the rGO-coated fabrics under harsh conditions demonstrate promising potentials for many practical applications.

To study and develop a health-monitoring system based on smart garments and wearable characteristics, the mechanical and electrical properties of rGO-coated fabric was investigated with a tensile test (Fig. 7). Pristine fabrics and three other kinds of prepared fabrics coated with rGO were characterized by a tensile test method ${ }^{18}$ carried out by using a micro-tester with a constant displacement rate of $2.5 \mu \mathrm{m} \mathrm{s}^{-1}$ and a gap between the clamps of $50 \mathrm{~mm}$. The resistance of the samples was recorded as the fracture elongation ratio was obtained (Fig. S5 $\dagger$ ). The pristine fabric's plastic deformation's maximum elongation ratio was $293.3 \%$ until failure and the rGO-coated fabrics exhibited an elastic deformation of $56.56 \%$ that avoided a large deformation for the maintenance of shape and functionality. The fabrics coated with rGO along with the MTIP process lasted six times longer than other samples without

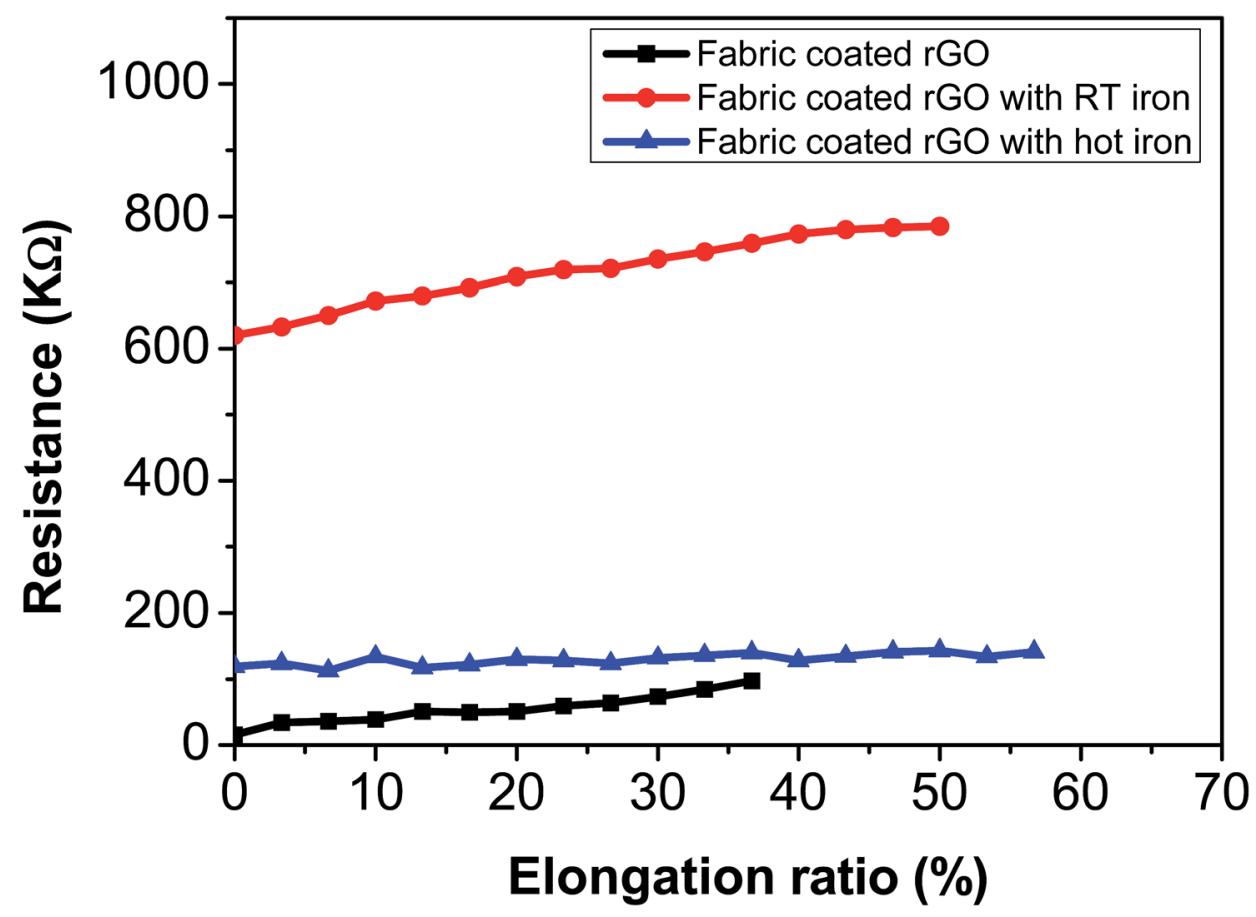

Fig. 7 Electrical properties of $\mathrm{rGO}$-coated fabric related to the elongation ratio. 
MTIP. Moreover, it was noteworthy that pristine fabric was an insulator, while rGO-coated fabrics became conductive due to the thermally reduced GO (Fig. 7 and S6†). As mentioned in the Experiment section, we sprayed the GO solution on the fabrics' surfaces. The hot iron reduced the GO into rGO coating on the fabrics more than that just using a room-temperature iron. The MTIP process highly diminished the resistance of the rGOcoated fabrics from more than 620 to around $110 \mathrm{k} \Omega$. However, a part of the GO coating was removed during the MTIP process when the iron was pressed on the fabrics, which resulted the fabric without MTIP process having the thickest rGO coating and the lowest resistance after the oven thermally reduction treatment. A highly conductive, flexible, and durable fabric was successfully produced for potential applications of smart cloth and wearable circuits, which are necessary for the future industry.

\section{Conclusions}

This study has demonstrated the simple and low-cost process based fabrication of robust, reusable, flexible, mechanically stable, hydrophobic, and oleophilic fabrics coated with rGO by just using GO solutions, fabrics, an iron, and an oven. The research for modifying the surface was simple and required no special equipment, which makes it suitable for large-scale production. The rGO fabrics revealed a hydrophobic surface and had a water contact angle of $\sim 129.4^{\circ}$ after thermal reduction. Low interfacial energy organic solvents and oil were freely filtered, but water did not permeate through the separation fabrics due to the hydrophobicity of the surface and the petal effect. The separation fabrics exhibited a stable performance in water-oil separation recycling. The MTIP process turned the pristine fabric from an insulator to a conductive material due to the thermally reduced GO. Furthermore, the rGO-coated fabrics displayed increased mechanical properties and robustness. This approach is beneficial for various applications which require high separation rates and low costs. For example, it could clean up ship oil spills in the ocean, where GO-coated fabrics could be used to drag out oil from contaminated seawater for reuse. These findings have significant implications for the understanding of the MTIP process. The anti-wetting, self-cleaning properties, and potential utilization of their electrical and mechanical properties for practical applications will meet the rapidly evolving demands for advanced multifunctional clothes.

\section{Acknowledgements}

This work was supported by the Center for Advanced Soft Electronics and Basic Science Research Program through the National Research Foundation (NRF), funded by the Ministry of Science, ICT and Future Planning (MSIP) in Korea.

\section{Notes and references}

1 I. P. Parkin and R. G. Palgrave, J. Mater. Chem., 2005, 15, 1689.
2 Y. Liu, J. Tang, R. Wang, H. Lu, L. Li, Y. Kong, K. Qi and J. Xin, J. Mater. Chem., 2007, 17, 1071.

3 L. Feng, Y. Zhang, J. Xi, Y. Zhu, N. Wang, F. Xia and L. Jiang, Langmuir, 2008, 24, 4114.

4 S. Kim, E. Cheung and M. Sitti, Langmuir, 2009, 25, 7196.

5 K. Koch, B. Bhushan and W. Barthlott, Progress in Materials Science, 2009, 54, 137.

6 X. Wang, B. Ding, J. Yu and M. Wang, Nano Today, 2011, 6, 510.

7 B. J. Sparks, E. F. T. Hoff, L. Xiong, J. T. Goetz and D. L. Patton, ACS Appl. Mater. Interfaces, 2013, 5, 1811.

8 Y.-C. Kim, K.-H. Lee, S. Sasaki, K. Hashimoto, K. Ikebukuro and I. Karube, Anal. Chem., 2000, 72, 3379.

9 C. S. Tian and Y. R. Shen, Proc. Natl. Acad. Sci. U. S. A., 2009, 106, 15148.

10 J. Wang, Y. Zhang, S. Wang, Y. Song and L. Jiang, Acc. Chem. Res., 2011, 44, 405.

11 X. Deng, L. Mammen, H.-J. Butt and D. Vollmer, Science, 2012, 335, 67.

12 M. T. Khorasani, H. Mirzadeh and P. G. Sammes, Radiat. Phys. Chem., 1996, 47, 881.

13 B. R. D'Urso and J. T. Simpson, Google Patents, 2007.

14 L. W. Hrubesh, P. R. Coronado and J. H. Satcher, J. NonCryst. Solids, 2001, 285, 328.

15 L. J. Chen, M. Chen, H. Di Zhou and J. M. Chen, Appl. Surf. Sci., 2008, 255, 3459.

16 R. K. Joshi, P. Carbone, F.-C. Wang, V. G. Kravets, Y. Su, I. V. Grigorieva, H. A. Wu, A. K. Geim and R. R. Nair, Science, 2014, 343, 752.

17 H. Qin, T. Gong, Y. Cho, C. Lee and T. Kim, Polym. Chem., 2014, 5, 4466.

18 T. Gong, D. V. Lam, R. Liu, S. Won, Y. Hwangbo, S. Kwon, J. Kim, K. Sun, J. H. Kim and S. M. Lee, Adv. Funct. Mater., 2015, 25, 3756.

19 D. Gao, H.-s. Cui, T.-t. Huang, D.-f. Yang and J.-x. Lin, J. Supercrit. Fluids, 2014, 86, 108.

20 D. R. Dreyer, S. Park, C. W. Bielawski and R. S. Ruoff, Chem. Soc. Rev., 2010, 39, 228.

21 G. Hayase, K. Kanamori, M. Fukuchi, H. Kaji and K. Nakanishi, Angew. Chem., Int. Ed., 2013, 52, 1986.

22 Z. Xue, S. Wang, L. Lin, L. Chen, M. Liu, L. Feng and L. Jiang, Adv. Mater., 2011, 23, 4270.

23 O. C. Compton and S. T. Nguyen, Small, 2010, 6, 711.

24 S. Pei and H.-M. Cheng, Carbon, 2012, 50, 3210.

25 J. Zhang, H. Yang, G. Shen, P. Cheng, J. Zhang and S. Guo, Chem. Commun., 2010, 46, 1112.

26 Y. Zhu, M. D. Stoller, W. Cai, A. Velamakanni, R. D. Piner, D. Chen and R. S. Ruoff, ACS Nano, 2010, 4, 1227.

27 Y. Zhu, S. Murali, W. Cai, X. Li, J. W. Suk, J. R. Potts and R. S. Ruoff, Adv. Mater., 2010, 22, 3906.

28 J. T. Robinson, S. M. Tabakman, Y. Liang, H. Wang, H. Sanchez Casalongue, D. Vinh and H. Dai, J. Am. Chem. Soc., 2011, 133, 6825.

29 K. Krishnamoorthy, M. Veerapandian, K. Yun and S. J. Kim, Carbon, 2013, 53, 38.

30 W. Zhang, Z. Shi, F. Zhang, X. Liu, J. Jin and L. Jiang, Adv. Mater., 2013, 25, 2071. 